University of Nebraska - Lincoln DigitalCommons@University of Nebraska - Lincoln

$1-25-2013$

\title{
Near-room-temperature refrigeration through voltage-controlled entropy change in multiferroics
}

Christian Binek

University of Nebraska-Lincoln, cbinek@unl.edu

V. Burobina

University of Nebraska-Lincoln

Follow this and additional works at: http://digitalcommons.unl.edu/physicsbinek

Binek, Christian and Burobina, V., "Near-room-temperature refrigeration through voltage-controlled entropy change in multiferroics" (2013). Christian Binek Publications. 80.

http://digitalcommons.unl.edu/physicsbinek/80

This Article is brought to you for free and open access by the Research Papers in Physics and Astronomy at DigitalCommons@University of Nebraska Lincoln. It has been accepted for inclusion in Christian Binek Publications by an authorized administrator of DigitalCommons@University of Nebraska - Lincoln. 


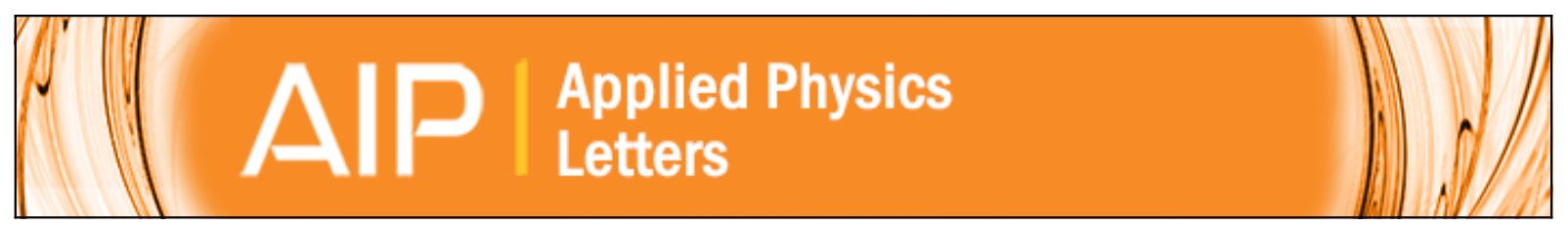

Near-room-temperature refrigeration through voltage-controlled entropy change in multiferroics

Ch. Binek and V. Burobina

Citation: Applied Physics Letters 102, 031915 (2013); doi: 10.1063/1.4788690

View online: http://dx.doi.org/10.1063/1.4788690

View Table of Contents: http://scitation.aip.org/content/aip/journal/apl/102/3?ver=pdfcov

Published by the AIP Publishing

\section{Articles you may be interested in}

Room-temperature single phase multiferroic magnetoelectrics: $\mathrm{Pb}(\mathrm{Fe}, \mathrm{M}) \mathrm{x}(\mathrm{Zr}, \mathrm{Ti})(1-\mathrm{x}) \mathrm{O} 3[\mathrm{M}=\mathrm{Ta}, \mathrm{Nb}]$

J. Appl. Phys. 113, 074105 (2013); 10.1063/1.4790317

Control of magnetism in $\mathrm{Pb}(\mathrm{ZrO} .2 \mathrm{Ti0} 0.8) \mathrm{O} 3 / \mathrm{La} 0.8 \mathrm{Sr} 0.2 \mathrm{MnO} 3$ multiferroic heterostructures (invited)

J. Appl. Phys. 109, 07D905 (2011); 10.1063/1.3540694

Room temperature multiferroic effects in superlattice nanocapacitors

Appl. Phys. Lett. 97, 252902 (2010); 10.1063/1.3528210

Temperature dependence of the magnetoelectric effect in $\mathrm{Pb}$ ( $\mathrm{Zr} 0.2 \mathrm{Ti} 0.8$ ) $\mathrm{O} 3$ / La 0.8 Sr 0.2 MnO 3 multiferroic heterostructures

Appl. Phys. Lett. 97, 042506 (2010); 10.1063/1.3472259

Giant magnetoelectric coupling and E -field tunability in a laminated Ni 2 MnGa /lead-magnesium-niobate-lead titanate multiferroic heterostructure

Appl. Phys. Lett. 93, 112502 (2008); 10.1063/1.2986480

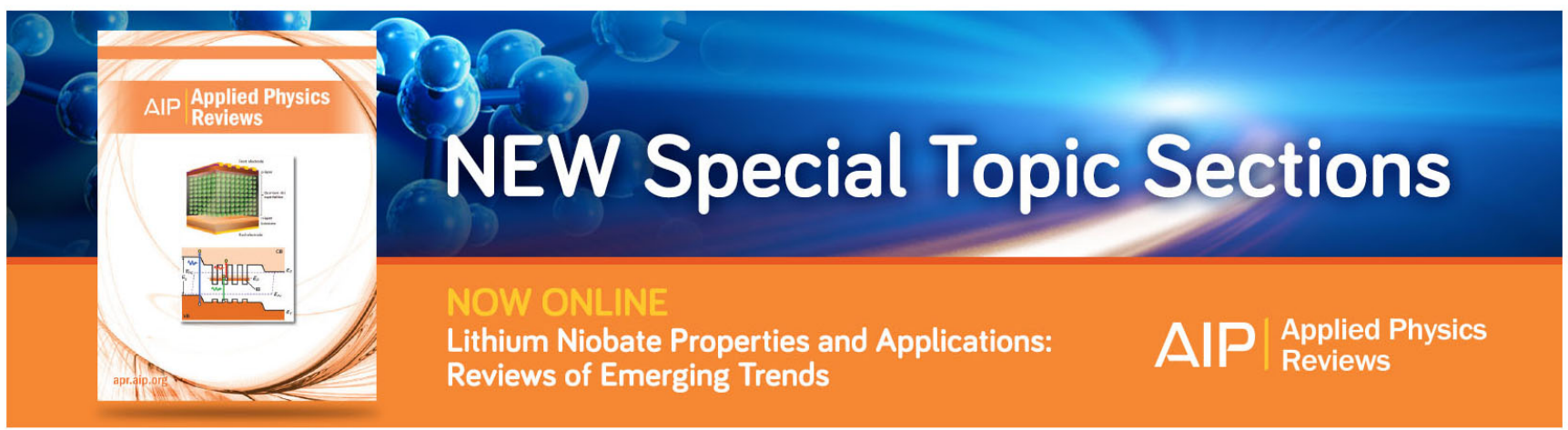




\title{
Near-room-temperature refrigeration through voltage-controlled entropy change in multiferroics
}

\author{
Ch. Binek ${ }^{\text {a) }}$ and V. Burobina \\ Department of Physics and Astronomy, Nebraska Center for Materials and Nanoscience, Theodore Jorgensen \\ Hall, 855 North 16th Street, University of Nebraska, P.O. Box 880299, Lincoln, Nebraska 68588-0299, USA
}

(Received 27 July 2012; accepted 7 January 2013; published online 25 January 2013)

\begin{abstract}
Composite materials with large magnetoelectric effect are proposed for application in advanced near-room-temperature refrigeration. The key innovation rests on utilizing the magnetocaloric effect in zero applied magnetic fields. This approach promises sizable isothermal entropy change and virtually temperature-independent refrigerant capacity through pure voltage-control. It is in sharp contrast with the conventional method of exploiting the magnetocaloric effect through applied magnetic fields. We outline the thermodynamics and estimate an isothermal entropy change specifically for the $\mathrm{La}_{0.7} \mathrm{Sr}_{0.3} \mathrm{MnO}_{3} / \mathrm{Pb}\left(\mathrm{Mg}_{1 / 3} \mathrm{Nb}_{2 / 3}\right) \mathrm{O}_{3}-\mathrm{PbTiO}_{3}(001)$ two-phase composite material. Finally, we propose structural variations of two-phase composites, which help in overcoming the challenging task of producing nanostructured material in macroscopic quantities. (C) 2013 American Institute of Physics. [http://dx.doi.org/10.1063/1.4788690]
\end{abstract}

Magnetic refrigeration has its roots in adiabatic demagnetization, utilized in the early days of low-temperature physics. In recent years, there has been revived interest in this technology. Advances in materials science make magnetic refrigeration near-room-temperature a promising approach towards an energy-efficient and environmentally friendly refrigeration technology. ${ }^{1-4}$ According to the DOE Energy Information Administration, heating, ventilation, and cooling (HVAC) accounted for $3856 \times 10^{9} \mathrm{~kW} \mathrm{~h}$, or $36 \%$ of the electricity consumed by U.S. households in 2011. Central air-conditioning and refrigeration alone accounted for $30 \%$ of the total electricity used in homes. Therefore, there is a clear consensus that improved refrigeration technology is of major importance and, potentially, a big part of the solution to the energy crisis.

The magnetocaloric effect (MCE) provides the physical basis of magnetic refrigeration technology which has the potential to replace today's common compression refrigerators. ${ }^{5-8}$ Recently, nanotechnology has enriched the quest for advance magnetocaloric materials utilizing nanocomposition for materials design. ${ }^{9-13}$ Despite the innovation in the field of magnetocaloric materials science through the nanotechnological approach, the scientific community kept looking at the problem from the conventional perspective of employing the MCE by exposing the active magnetocaloric material to an external magnetic field.

The isothermal entropy change and the adiabatic temperature change are two of the most important parameters that characterize and quantify the MCE. ${ }^{14}$ An integrated Maxwell relation determines the isothermal entropy change, $\Delta S$. Most researchers refer to the Maxwell relation which originates from the analytic properties of the Gibbs free energy. It reads

$$
\Delta S=\mu_{0} V \int_{H_{i}}^{H_{f}}\left(\frac{\partial M}{\partial T}\right)_{H} d H,
$$

where $H_{i, f}$ are the initial (typically zero) and final applied magnetic field, $M$ is the magnetization, $V$ is the volume of the active magnetocaloric material, and $\mu_{0}$ is the vacuum permeability. Equation (1) is a rigorous and fundamental expression valid in all situations where the mixed secondorder derivatives of the Gibbs free energy exist and the order of differentiation can be exchanged. This is, in general, the case, with the exception of first-order phase transitions, where the entropy change at the transition can be calculated with the help of the Clausius-Clapeyron equation. ${ }^{9,15}$

Equation (1) misleadingly suggests that the use of an external magnetic field is mandatory in order to achieve a sizeable isothermal entropy change. It is one of the major objectives of this manuscript to offer evidence that applied magnetic fields are not necessary to utilize the MCE. In addition, it is our goal to highlight the fact that pure voltageinduced entropy change in magnetocaloric materials has significant advantages over conventional magnetic fieldinduced entropy change with the potential to revolutionize magnetic refrigeration technology.

It is the focus on magnetic field-induced caloric effects which completely dominates past and ongoing efforts in the quest for optimized magnetocaloric materials. In order to bring magnetocaloric refrigeration technology to a level where its figure of merit is outperforming conventional refrigeration appliances, one needs to overcome this limitation. Particularly, the need for magnetic fields and the difficulty to provide them with significant strength is hampering progress. Although the investigation of the electrocaloric effect is rapidly progressing, ${ }^{16}$ there are still large challenges from the materials perspective. It is therefore beneficial to combine the advantages of magnetocaloric and electrocaloric materials by utilizing the MCE through pure voltage control.

Before we describe the feasibility of this approach, we will highlight a few advantages of the voltage-controlled entropy change over the traditional method employing applied magnetic fields. The ordinary path towards sizable isothermal entropy change relies on applying magnetic fields to ever higher final values, $H_{\mathrm{f}}$, until technical saturation of the

\footnotetext{
${ }^{a}$ Electronic mail: cbinek@unl.edu.
} 
magnetization is reached. This brute-force approach has practical limitations. When relying on the maximum achievable flux densities of 1-2 $\mathrm{T}$ of modern permanent magnets ${ }^{17,18}$ (Nd-Fe-B or Sm-Co), the feasible adiabatic temperature changes remain below $10 \mathrm{~K} .{ }^{19,20}$ Permanent magnetic flux densities of the order of $4 \mathrm{~T}$ can be created in Halbach cylinders, but logarithmic dependence of the field on the diameter of the cylinder makes such devices very heavy. ${ }^{18}$ Since permanent magnets are the only option to create magnetic fields in an energy efficient manner, all of today's realizations of magnetocaloric refrigerators have to utilize a mechanism which moves the magnetocaloric material relative to the permanent magnet in order to create a sizable change in magnetic field, $\Delta H=H_{f}-H_{i}$. The need of moving parts is something that should be avoided since it creates noise, losses in friction, and wear-and-tear of components.

All of these disadvantages of magnetic field-induced entropy changes can be avoided when employing voltagecontrolled entropy change in the absence of electric currents. Next, the mechanism of voltage-activated MCE is outlined.

One well-known branch of voltage-control of magnetic properties utilizes piezoelectrically induced strain to control anisotropy and critical temperature. For example, piezoelectrically induced strain is used to substantially change the magnetic anisotropy in Fe thin films or exploited to control the exchange-bias field in an exchange-bias magnetic heterostructure. ${ }^{21-24}$ Similarly, and of major significance here, strain originating from stress can be induced via the inverse piezoelectric effect. The strain, when carried over into an adjacent magnetic thin film, can substantially change the magnetic Curie temperature, $T_{\mathrm{C}}$. An external control parameter such as tensile or compressive strain can tune the degree of magnetic order in a magnetically long-range ordered system. Decrease (increase) in long-range magnetic order can be accompanied by a significant increase (decrease) in entropy. In particular, the transition from a paramagnetic into a longrange ordered magnetic state is accompanied by sizeable entropy reduction.

It has been shown by Angeloni et al. that a compressively strained $\mathrm{La}_{0.7} \mathrm{Sr}_{0.3} \mathrm{MnO}_{3}$ (LSMO) film of $16 \mathrm{~nm}$ thickness on $\mathrm{LaAlO}_{3}(001)$ changes the Curie temperature of the former from $365 \mathrm{~K}$ (unstrained) to $270 \mathrm{~K}$ (strained through lattice mismatch). ${ }^{25}$ Thiele et al. have shown that voltagecontrolled epitaxial strain in LSMO is possible when exploiting the inverse piezoelectric effect of $\mathrm{Pb}\left(\mathrm{Mg}_{1 / 3} \mathrm{Nb}_{2 / 3}\right) \mathrm{O}_{3^{-}}$ $\mathrm{PbTiO}_{3}(001)$ (PMN-PT) substrates. ${ }^{26}$ They report on voltage-controlled changes of the Curie temperature in a LSMO film of $20 \mathrm{~nm}$ thickness between $278 \mathrm{~K}$ and $297 \mathrm{~K}$. Fig. 1 displays the effect for the $20 \mathrm{~nm}$ thick LSMO sample reported in Ref. 27. Note that various parameters allow finetuning of the critical temperature at zero applied voltage. Those tuning parameters are the $\mathrm{Sr}$ concentration of the LSMO compound, the film thickness, and the initial strain in zero-applied electric field.

From these results, it is apparent that changes of the critical temperature, $T_{\mathrm{C}}$, of ferromagnetic thin films such as the complex oxide LSMO can be achieved by pure voltagecontrol when harnessing the inverse piezoelectric effect in order to strain the film in an electrically controlled manner.

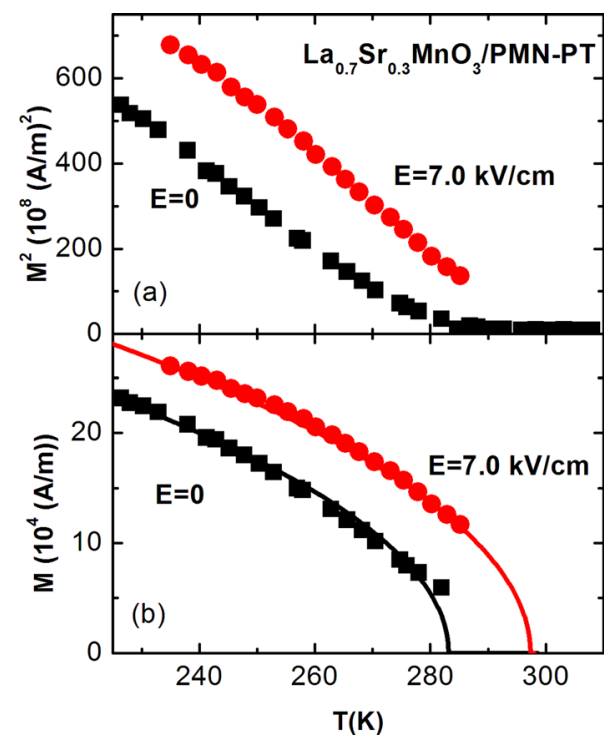

FIG. 1. (a) $M^{2}$ vs. $T$ representation of magnetization data of a LSMO $(20 \mathrm{~nm}) / \mathrm{PMN}-\mathrm{PT}$ for zero (squares) and $7.0 \mathrm{kV} / \mathrm{cm}$ (circles) applied electric field. (b) Linear plot $M$ vs. $T$ (squares $E=0$, circles $E=7 \mathrm{kV} / \mathrm{cm}$ ) together with best fits of the Landau-type functional form of the magnetization (lines). ${ }^{27}$

In addition, large magnetoelectric susceptibilities can also be achieved especially in complex oxides when utilizing the electric field effect, which mimics chemical doping. Recently, we demonstrated sizable electric modulation of magnetization in a $\mathrm{BaTiO}_{3} / \mathrm{LSMO}$ heterostructure. Here, the electric field-controlled metal-insulator transition in the LSMO film gives rise to a large magnetoelectric effect. ${ }^{28}$ It can be potentially exploited in the same way as the straininduced change in magnetization. Next, we outline thermodynamic consequences of large magnetoelectric effects and their impact on voltage-controlled isothermal entropy change.

We start from the differential form of the Helmholtz free energy, $F$, of a magnetic system

$$
d F=-S d T+\mu_{0} V H d M .
$$

We consider the hitherto unexplored case of the applied magnetic field being zero where $H$ in Eq. (2) is the internal magnetic field. The magnetization, in turn, is a function of a control parameter, which here is the electric field $E$, in accordance with Fig. 1. Therefore, the equation of state can be written as

$$
H=H(T, M(E)) .
$$

From Eq. (2), we derive a Maxwell relation by identifying the mixed second-order derivatives of the Helmholtz free energy

$$
\left(\frac{\partial S}{\partial M}\right)_{T}=-\mu_{0} V\left(\frac{\partial H}{\partial T}\right)_{M} .
$$


Integration of Eq. (4) provides the expression

$$
\Delta S=-\mu_{0} V \int_{M_{i}}^{M_{f}}\left(\frac{\partial H}{\partial T}\right)_{M} d M
$$

for the isothermal entropy change. Note that Eq. (5) differs from the most frequently used Eq. (1), which is derived from a different Maxwell relation originating from the Gibbs free energy.

Using thermodynamic identities, we obtain from Eq. (5)

$$
\Delta S=\int_{M_{i}}^{M_{f}} \frac{\mu_{0} V}{\chi}\left(\frac{\partial M}{\partial T}\right)_{H} d M
$$

where $\chi=\left(\frac{\partial M}{\partial H}\right)_{T}$ is the magnetic susceptibility. From the fact that $M$ depends on the control parameter, $E$, we obtain

$$
\Delta S=\int_{E_{i}=0}^{E_{f}} \frac{\mu_{0} V}{\chi}\left(\frac{\partial M}{\partial T}\right)_{H}\left(\frac{\partial M}{\partial E}\right)_{T} d E .
$$

To further explore the consequences of Eq. (7), we consider the Landau expression $F=F_{0}(T)+\frac{1}{2} A M^{2}+\frac{1}{4} B M^{4}$ $-\mu_{0} V H M$. Here, $A=a_{0}\left(T-T_{C}(E)\right), a_{0}$ and $B$ are positive constants, and $F_{0}$ is a regular temperature-dependent background. The Landau expression allows specifying $M$ and $\chi$ in terms of the expansion coefficients. This yields the entropy change for $T \leq T_{\mathrm{C}}(0)$

$$
\Delta S=-\frac{a_{0}^{2}}{2 B}\left(T_{C}(E)-T_{C}(0)\right) .
$$

One can replace the parameters of the Landau expansion using $\frac{a_{0}}{B}=\frac{M^{2}(T=0)}{T_{c}}$ and $\frac{\mu_{0} V}{\chi\left(2 T_{c}\right)}=a_{0} T_{C}$ which yields

$$
\Delta S=-\frac{\mu_{0} V M^{2}(T=0)}{2 \chi\left(2 T_{c}\right) T_{c}^{2}}\left(T_{C}(E)-T_{C}(0)\right) .
$$

Using a density value for $\mathrm{LSMO}^{29}$ of $\rho=6600 \mathrm{~kg} / \mathrm{m}^{3}$, we calculate a mass-specific isothermal entropy change which can now be used for comparison with current state-of-the-art magnetic field-induced, specific entropy changes. A literature value for the saturation magnetization ${ }^{30}$ of a pulsed laser deposited LSMO film of $30 \% \mathrm{Sr}$ concentration reads $M(T=0) \approx 0.45 \mathrm{MA} / \mathrm{m}$. Since susceptibility investigations of LSMO films are scarce and there is currently no reliable data basis, we use the mean-field expression ${ }^{31}$

$$
\frac{\mu_{0} M(0)}{\chi(T)}=\frac{3 k_{B} T_{C}}{g \mu_{B}(S+1)}\left(\frac{T}{T_{C}}-1\right),
$$

which for $T=2 T_{\mathrm{C}}$ yields $\chi\left(2 T_{c}\right)=\frac{\mu_{0} g \mu_{B}(S+1) M(0)}{3 k_{B} T_{C}}$, where the authors in Ref. 31 find experimentally $S \approx 3.5$. Using further a Landé $g$-factor of $g \approx 2$ in accordance with Ref. 32, $T_{\mathrm{C}}(E=0)=279 \mathrm{~K}$ and $\Delta T_{C}=T_{C}(E=7 \mathrm{kV} / \mathrm{cm})-T_{C}(0)=$ $19 \mathrm{~K}$, we estimate the value of the specific entropy change which yields $\Delta S / m=-1.15 \frac{\mathrm{J}}{\mathrm{kgK}}$.
Note that $S(T, E)$ and, therefore, Eq. (8) can be directly calculated from the Landau free energy according to $S=-\left(\frac{\partial F}{\partial T}\right)_{M}$. It yields

$$
\begin{aligned}
S(T, E) & =-\left(\frac{\partial F}{\partial T}\right)_{M}=-\frac{1}{2} \frac{\partial A}{\partial T} M^{2}+S_{0} \\
& = \begin{cases}-\frac{a_{0}^{2}}{2 B}\left(T_{C}(E)-T\right)+S_{0} & \text { for } T<T_{C}(E) \\
S_{0} & \text { for } T>T_{C}(E)\end{cases}
\end{aligned}
$$

Note, however, that Eq. (11) does not render Eq. (7) obsolete. From an experimental point of view, only Eq. (7) is valuable. First, it is free from approximations. Second, $\chi$ and $M(T, E)$ can be measured while the Landau free energy is not only a crude approximation of the underlying thermodynamic potential $F$ but, in addition, is not experimentally accessible.

Alternatively, we estimate the mass-specific isothermal entropy change at $T=280 \mathrm{~K}$ from the magnetoelectric susceptibility, $\alpha=\mu_{0} \frac{\partial M}{\partial E}$, of PMN-PT/LSMO. The numerical value of $\alpha$ is determined from the data in Fig. 1 (adapted from Ref. 27). For $E=0$ and $7 \mathrm{kV} / \mathrm{cm}$, the magnetization data are well described by the functions $M(T, E=0)=10^{4}$ $\sqrt{\left(2.63 \times 10^{3}-9.27 T / K\right)} \frac{A}{m}$ and $M(T, E=7 \mathrm{kV} / \mathrm{cm})=10^{4}$ $\sqrt{\left(3.24 \times 10^{3}-10.89 T / K\right)} \frac{A}{m}$ which yields $\alpha(T=280 K) \approx$ $\mu_{0}(M(T=280 K, E=7 \mathrm{kV} / \mathrm{cm})-M(T=280 K, E=0)) /(7.0$ $\left.\times 10^{5} \mathrm{~V} / \mathrm{m}\right)=2.41 \times 10^{-7} \frac{\mathrm{s}}{\mathrm{m}}$. We use this value of magnetoelectric susceptibility for further estimates and neglect the details of the temperature dependence of $\alpha$. This approximation is reasonable because the magnetoelectric susceptibility is virtually constant for $T<280 \mathrm{~K}$ where the $M$ vs. $T$ data sets (Fig. 1, squares and circles) are virtually parallel. In addition, we use a rough estimate for $M(0)=0.51 \times 10^{6} \frac{\mathrm{A}}{\mathrm{m}}$ for LSMO by extrapolating $M(T, E=0)$ towards $T=0$. The extrapolation of the Landau expression overestimates the magnetization at $T=0$ which, in turn, gives rise to an underestimation of the isothermal entropy change. We obtain $\left(\frac{\partial M}{\partial T}\right)_{T=280 K, E=0}=-8.53 \frac{\mathrm{kA}}{\mathrm{mK}}$ from the $M(T, E=0)$ function. In the absence of additional $M$ vs. $T$ data for $0<E<7 \mathrm{kV} / \mathrm{cm}$, we assume a linear dependence of $T_{\mathrm{C}}$ on the applied electric field which reads $T_{C}(E)=2.43 \times 10^{-5} \mathrm{EKm} / \mathrm{V}+280 \mathrm{~K}$. Using Eq. (10) to quantify $\chi\left(T, T_{C}(E)\right)$, we estimate the mass-specific isothermal entropy change from

$$
\Delta S / m=\int_{0}^{E} \frac{\alpha}{\chi\left(T, T_{C}\right) \rho}\left(\frac{\partial M}{\partial T}\right)_{H} d E .
$$

It yields $\Delta S / m=-1.43 \frac{\mathrm{J}}{\mathrm{kgK}}$ in good agreement with the alternative approach based on Eq. (9) and outlined above. The remaining difference in the numerical values of the specific isothermal entropy changes originates from differences in the assumptions and approximations. 
The voltage-induced entropy change estimated above is of respectable magnitude when compared, e.g., with the bulk giant magnetocaloric material $\mathrm{Gd}_{5} \mathrm{Si}_{2} \mathrm{Ge}_{2}$, which represents a benchmark for magnetocaloric materials. ${ }^{33} \mathrm{Gd}_{5} \mathrm{Si}_{2} \mathrm{Ge}_{2}$ has an isothermal entropy change of approximately $4 \mathrm{~J} / \mathrm{kg} \mathrm{K}$ when an external magnetic field is ramped from zero to $1 \mathrm{~T}$. Although this value is still approximately twice the entropy change, we estimate for the voltage-controlled effect in PMN-PT/LSMO, it is important to realize that for virtually all magnetocaloric materials and $\mathrm{Gd}_{5} \mathrm{Si}_{2} \mathrm{Ge}_{2}$, in particular, the isothermal entropy change strongly peaks at a given temperature, $T_{\max }$, and decreases for both higher and lower temperatures. This limits the refrigerant capacity, RC which is the true figure-of-merit of a refrigerator. $\mathrm{RC}$ can be calculated from the $T$-dependence of the isothermal entropy change according to $R C=\int_{T_{\max }-\Delta T / 2}^{T_{\max }+\Delta T / 2} \Delta S d T$. Here $\Delta T$ is the width of $\Delta S(T)$ at half-maximum. Therefore, for the narrow Gaussian $\Delta S$ vs. $T$ behavior, RC is limited despite potentially large values of $S$ at the maximum of $\Delta S$ vs. $T .^{34}$

In the case of voltage-controlled entropy change, $\Delta S$ vs. $T$ will remain virtually constant for all $T<T_{\mathrm{C}}(0)$ at the value given by Eq. (9). The absolute value of the entropy change decreases linearly to zero for $T>T_{\mathrm{C}}(0)$ and remains zero for $T \geq T_{\mathrm{C}}(E)$. Fig. 2 shows the $T$-dependences of $S(T, E=0)$ (circles), $S\left(T, E=E_{\mathrm{f}}>0\right)$ (squares) and $S\left(T, E=E_{\mathrm{f}}>0\right)$ $S(T, E=0)$ (right axis) as given by Eq. (11). The temperature independence of $\Delta S$ vs. $T$ for the temperatures $T<T_{\mathrm{C}}(0)$ largely increases the RC to values potentially much higher than those reported in the literature. Note, however, that the emphasis here is not so much on overcoming the entropy change and RC of the best magnetocaloric materials, but to achieve sizeable entropy change in the absence of applied magnetic fields. Pure voltage-controlled entropy change broadens the range of potential applications when compact cooling solutions with little to no mechanical vibrations are required.

The bilayer system PMN-PT/LSMO can be considered a generic and prototypical building block of a two-phase multiferroic ${ }^{35}$ material, ${ }^{36}$ allowing for voltage-controlled entropy change. In general, composite materials combining a piezoelectric material with a magnetic material of sizeable

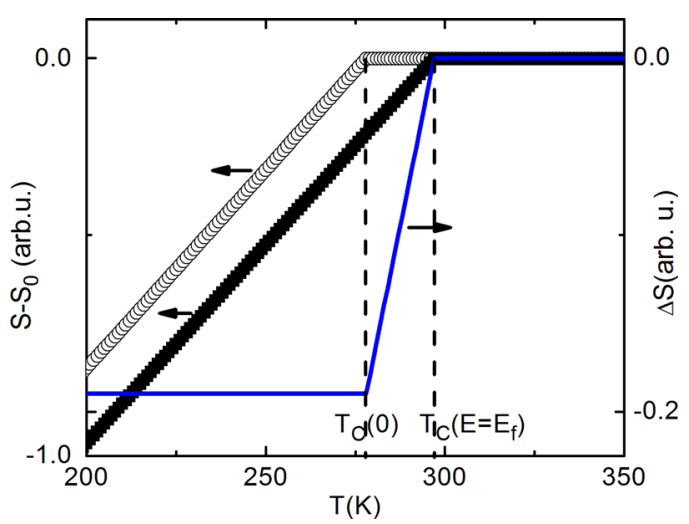

FIG. 2. Sketch of the temperature dependence of the entropy for $E=0$ (circles) and $E=E_{\mathrm{f}}>0$ (squares) according to Eq. (11). The line (right axis) shows the temperature dependence of the isothermal entropy change. $T_{\mathrm{C}}(0)$ and $T_{\mathrm{C}}\left(E=E_{\mathrm{f}}\right)$ are the critical temperatures (dashed lines) of the ferromagnetic film in electric fields $E=0$ and $E=\mathrm{E}_{\mathrm{f}}$, respectively. magnetoelastic response or other sources of large magnetoelectric response in multiferroic systems ${ }^{28}$ near room temperature are capable of functioning as a potential candidate for voltage-controlled entropy change. Another candidate of a magnetoelectric composite for magnetocaloric applications is a laminate composite of piezoelectric AlN and amorphous $\mathrm{FeCoSiB}$ which can be fabricated via sputtering methodology and has an extremely high magnetoelectric effect at room temperature. ${ }^{37}$ Operation of the laminate in ac mode at resonance frequency and in the presence of a small biasing magnetic field (order of the Earth's magnetic field) gives rise to $\alpha \approx 1.4 \times 10^{-7} \mathrm{~s} / \mathrm{m}$ which may prove suitable as an alternative to the complex oxide composites analyzed here in more detail. In addition, the $M$-type hexaferrite $\mathrm{SrCo}_{2} \mathrm{Ti}_{2-}$ $\mathrm{Fe}_{8} \mathrm{O}_{19}$ shows a promising large magnetoelectric effect at room temperature which is about 50 times higher than the maximum magnetoelectric susceptibility $\alpha \approx 4 \times 10^{-12} \mathrm{~s} / \mathrm{m}$ of the archetypical magnetoelectric chromia. ${ }^{38,39}$ This bulk magnetoelectric susceptibility is still orders of magnitude below the magnetoelectric response of LSMO and $\mathrm{FeCoSiB}$ composites. Nevertheless, it indicates that new bulk magnetoelectric multiferroics may become alternatives to the suggested composite materials especially when considering that bulk materials overcome the complications which accompany the production of large quantities of nanofabricated materials.

For applications in magnetocaloric refrigeration, macroscopic amounts of active material are required. We suggest several fabrication strategies which are depicted in Fig. 3. These include mixtures of grains of piezoelectric and magnetoelastic materials (Fig. 3(a)), thin film heterostructures with a large number of repetitions of the bilayer building block (Fig. 3(b)), or various ordered nanostructured arrangements such as nanopillars of magnetoeleastic material in a piezoelectric matrix (Fig. 3(c)) or vice versa (Fig. 3(d)). Here, the optimum structural choice will be affected by additional constraints such as optimized thermal conductivity. ${ }^{7}$

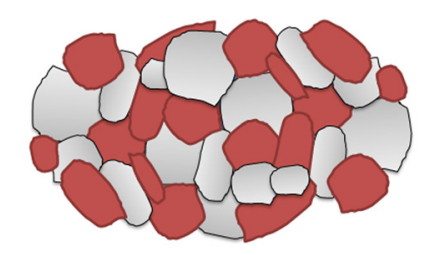

(a)

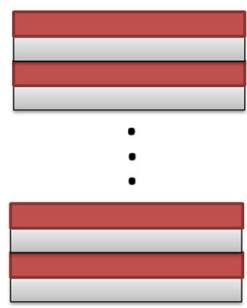

(b)

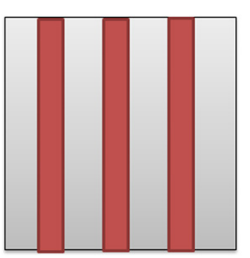

(c)

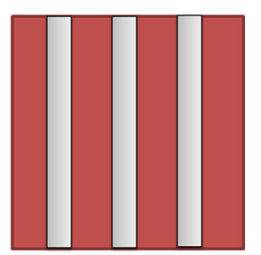

(d)
FIG. 3. Suggested nanofabrication of magnetocaloric materials for voltagecontrolled entropy change. Magnetic material (light gray) with magnetoeleastic properties such as LSMO interacts with piezoelectric material (dark gray/on-line red) such as PMN-PT when brought in close proximity. Possible nanostructured two-phase multiferroics can have grain structure (a), layered heterostructures (b), columnar structure of piezoelectric material in magnetic matrix (c), or vice versa (d). 
The concept of voltage-controlled entropy change in magnetocaloric materials for magnetic refrigeration applications has been introduced. The key innovation of this approach is based on the fact that the magnetocaloric effect is utilized without applying an external magnetic field. Using various experimental data available from the literature, we estimate a specific isothermal voltage-controlled entropy change for the bilayer heterostructure PMN-PT/LSMO. It is larger than $1 \mathrm{~J} / \mathrm{kg} \mathrm{K}$ and serves as proof of principle for voltage-controlled magnetic refrigeration close to room temperature. Various parameters to fine-tune the effect have been identified. An unusually large refrigerant capacity has been identified as a generic property of voltage-controlled magnetocaloric multiferroics. Four schemes have been suggested which allow one to produce macroscopic amounts of nanostructured magnetocaloric material. Potential benefits of this approach have been discussed and compared with the current method to induce entropy change in magnetocaloric materials with applied magnetic fields.

${ }^{1}$ V. K. Pecharsky and K. A. Gschneidner, Jr., Int. J. Refrig. 29, 1239 (2006).

${ }^{2}$ V. K. Pecharsky and K. A. Gschneidner, Jr., J. Magn. Magn. Mater. 200, 44 (1999)

${ }^{3}$ S. L. Russek and C. Zimm, Int. J. Refrig. 29, 1366 (2006).

${ }^{4}$ K. A. Gschneidner, Jr., V. K. Pecharsky, and A. O. Tsokol, Rep. Prog. Phys. 68, 1479 (2005).

${ }^{5}$ C. Zimm, A. Boeder, J. Chell, A. Sternberg, A. Fujita, S. Fujieda, and K. Fukamichi, Int. J. Refrig. 29, 1302 (2006).

${ }^{6}$ M. A. Richard, A. M. Rowe, and R. Chahine, J. Appl. Phys. 95, 2146 (2004).

${ }^{7}$ X. Bohigas, E. Molins, A. Roig, J. Tejada, and X. X. Zhang, IEEE Trans. Magn. 36, 538 (2000)

${ }^{8}$ L. Chen and Z. Yan, J. Appl. Phys. 75, 1249 (1994).

${ }^{9}$ T. Mukherjee, S. Sahoo, R. Skomski, D. J. Sellmyer, and Ch. Binek, Phys. Rev. B 79, 144406 (2009).

${ }^{10}$ T. Mukherjee, S. Michalski, R. Skomski, D. J. Sellmyer, and Ch. Binek, Phys. Rev. B 83, 214413 (2011).

${ }^{11}$ S. Michalski, R. Skomski, T. Mukherjee, X.-Zh. Li, Ch. Binek, and D. J. Sellmyer, J. Appl. Phys. 109, 07A936 (2011).

${ }^{12}$ R. Skomski, Ch. Binek, S. Michalski, T. Mukherjee, A. Enders, and D. J. Sellmyer, J. Appl. Phys. 107, 09A922 (2010).

${ }^{13}$ D. D. Belyea, T. S. Santos, and C. W. Miller, J. Appl. Phys. 111, 07A935 (2012).

${ }^{14}$ M. E. Wood and W. H. Potter, Cryogenics 25, 667 (1985).
${ }^{15}$ T. Mukherjee, R. Skomski, S. Michalski, D. J. Sellmyer, and Ch. Binek, J. Appl. Phys. 111, 07A931 (2012).

${ }^{16}$ J. F. Scott, Annu. Rev. Mater. Res. 41, 229 (2011).

${ }^{17}$ D. J. Sellmyer and R. Skomski, Advanced Magnetic Nanostructures (Springer 2006).

${ }^{18}$ R. Skomski and J. M. Coey, Permanent Magnetism (Institute of Physics, Bristol, 1999).

${ }^{19}$ H. Wada and Y. Tanabe, Appl. Phys. Lett. 79, 3302 (2001).

${ }^{20}$ V. K. Pecharsky and K. A. Gschneidner, Jr., Phys. Rev. Lett. 78, 4494 (1997).

${ }^{21}$ S. Sahoo, S. Polisetty, C.-G. Duan, S. S. Jaswal, E. Y. Tsymbal, and Ch. Binek, Phys. Rev. B 76, 092108 (2007).

${ }^{22}$ S. Polisetty, W. Echtenkamp, K. Jones, X. He, S. Sahoo, and Ch. Binek, Phys. Rev. B 82, 134419 (2010).

${ }^{23}$ X. He, Y. Wang, N. Wu, A. N. Caruso, E. Vescovo, K. D. Belashchenko, P. A. Dowben, and Ch. Binek, Nature Mater.9, 579 (2010).

${ }^{24}$ P. Borisov, A. Hochstrat, X. Chen, W. Kleemann, and Ch. Binek, Phys. Rev. Lett. 94, 117203 (2005).

${ }^{25}$ M. Angeloni, G. Balestrino, N. G. Boggio, P. G. Medaglia, P. Orgiani, and A. Tebano, J. Appl. Phys. 96, 6387 (2004).

${ }^{26}$ C. Thiele, K. Dörr, S. Fähler, L. Schultz, D. C. Meyer, A. A. Levin, and P. Paufler, Appl. Phys. Lett. 87, 262502 (2005).

${ }^{27}$ C. Thiele, K. Dörr, O. Bilani, J. Rödel, and L. Schultz, Phys. Rev. B 75, 054408 (2007).

${ }^{28}$ H. Lu, T. A. George, Y. Wang, I. Ketsman, J. D. Burton, C.-W. Bark, S. Ryu, D. J. Kim, J. Wang, C. Binek, P. A. Dowben, A. Sokolov, C.-B. Eom, E. Y. Tsymbal, and A. Gruverman, Appl. Phys. Lett. 100, 232904 (2012).

${ }^{29}$ T. J. Armstrong and A. V. Virkar, J. Electrochem. Soc. 149, A1565 (2002).

${ }^{30}$ M. Ziese, I. Vrejoiu, A. Setzer, A. Lotnyk, and D. Hesse, New J. Phys. 10, 063024 (2008).

${ }^{31}$ M. B. Salamon and S. H. Chun, Phys. Rev. B 68, 014411 (2003).

${ }^{32}$ H. P. Yang, L. Shi, S. M. Zhou, J. Y. Zhao, L. F. He, and Y. B. Jia, J. Phys.: Condens. Matter 21, 046002 (2009).

${ }^{33}$ A. O. Pecharsky, K. A. Gschneidner, and V. K. Pecharsky, J. Appl. Phys. 93, 4722 (2003).

${ }^{34}$ V. Provenzano, A. J. Shapiro, and R. D. Shull, Nature 429, 853 (2004).

${ }^{35}$ In a strict sense, one would refer to a material only as multiferroic if two ferroic order parameters such as magnetization and ferroelectric polarization are simultaneously present. Here, we use this term from a broader perspective. The piezoelectric materials can be a ferroelectric material, since all ferroelectrics are piezoelectric. A non-ferroelectric but piezoelectric material combined with a ferromagnetic material, however, is considered here as well a two-phase multiferroic.

${ }^{36}$ W. Eerenstein, N. D. Mathur, and J. F. Scott, Nature 442, 759 (2006).

${ }^{37}$ J. Ma, J. Hu, Z. Li, and C.-W. Nan, Adv. Mater. 23, 1062 (2011).

${ }^{38}$ L. Wang, D. Wang, Q. Cao, Y. Zheng, H. Xuan, J. Gao, and Y. Du, Sci. Rep. 2, 223 (2012).

${ }^{39}$ A. P. Pyatakov and A. K. Zvezdin, Phys. Usp. 55, 557 (2012). 